\title{
Téoros
}

Revue de recherche en tourisme

\section{Tourisme sexuel et virtualité} Le voyage dans le cyberespace

\section{Louis-Robert Frigault}

Volume 22, numéro 1, printemps 2003

Tourisme et sexualité

URI : https://id.erudit.org/iderudit/1071579ar

DOI : https://doi.org/10.7202/1071579ar

Aller au sommaire du numéro

Éditeur(s)

Université du Québec à Montréal

ISSN

0712-8657 (imprimé)

1923-2705 (numérique)

Découvrir la revue

Citer cet article

Frigault, L.-R. (2003). Tourisme sexuel et virtualité : le voyage dans le cyberespace. Téoros, 22(1), 10-14. https://doi.org/10.7202/1071579ar d'utilisation que vous pouvez consulter en ligne.

https://apropos.erudit.org/fr/usagers/politique-dutilisation/ 


\title{
Tourisme sexuel et virtualité
}

\author{
Le voyage dans le cyberespace
}

\section{Louis-Robert Frigault}

$\Delta$ ssocier aujourd'hui les concepts de tourisme et de sexualité nous plonge dans un imaginaire fécond où les pays d'Asie, d'Amérique du Sud ou d'Europe de l'Est se présentent comme des refuges pour des hommes à la recherche d'enfants ou de femmes à des fins strictement sexuelles. Dans cette perspective, le regard touristique en tant que processus de consommation des lieux (Urry, 1995) se transforme dans le cas du tourisme sexuel en un processus de consommation sexuelle d'individus. Nombreux sont les travaux qui ont mis en évidence - et surtout dénoncé - de telles pratiques (Hughes, 1996). Aussi, des organismes de lutte pour les droits de la personne et des enfants ont-ils réclamé l'adoption de législations très musclées pour mettre en échec le commerce international du sexe des enfants (UNICEF, 2001). Certains attribuent au réseau Internet une grande part de la responsabilité de l'augmentation du tourisme sexuel. Il constituerait un lieu efficace pour échanger de l'information sur les pays dont les législations sont les plus laxistes en matière de régulation du commerce sexuel, y compris celui des enfants. De plus, sa faible régulation sur le plan international permettrait à certains promoteurs de proposer leurs services sur Internet en toute impunité, participant ainsi au maintien et à l'expansion du phénomène.

Outre la croissance fulgurante de l'utilisation d'Internet par les agences de tourisme et les offices de tourisme pour promouvoir les attraits « légitimes » de leur pays, on voit apparaître de nombreux sites Internet où des individus, constitués en communautés vir- tuelles, échangent de l'information sur des thèmes aussi variés que les lieux fréquentés par les prostituées et les entremetteurs, les modalités de rencontre (codes sociaux et culturels), les actes sexuels acceptés et même les tarifs exigés pour certains services sexuels particuliers. Ces communautés virtuelles se constituent comme des espaces de partage et d'échange où les histoires sexuelles vécues en situation touristique occupent une place très importante.

La mise en place d'Internet, un réseau décentralisé d'ordinateurs permettant l'échange d'information touchant à peu près tous les domaines, a profondément transformé les modes de communication en créant un cyberespace aux frontières floues qui remplit des fonctions variées desquelles se dégagent des usages multiples : communiquer, apprendre, explorer et rencontrer. Au moment d'écrire ces lignes, on annonce que $54 \%$ de la population québécoise se sert d'Internet au moins une fois par semaine (NETendances, 2003). Internet n'est plus, du moins dans les sociétés occidentales, un objet réservé à une petite élite ou à des universitaires. Aussi, considérant le développement des usages sociaux d'Internet, on pourrait penser que si le cyberespace est un espace déterritorialisé, il constitue un nouveau lieu de circulation qui nous invite à repenser les modalités contemporaines du tourisme en général et du tourisme sexuel en particulier.

Nous proposerons dans cet article une réflexion sur le tourisme, la sexualité et Internet selon deux axes. Le premier permettra de dégager et d'illustrer la manière dont Internet faciliterait l'échange d'information sur le tourisme sexuel et parti- ciperait à son expansion. Le second s'intéressera au cyberespace comme un territoire complexe dans lequel on navigue ou sur lequel on « surfe », ce qui nous permet de repenser les liens entre le tourisme, la sexualité et Internet. En analysant les déplacements (du clic de la souris) dans les espaces sexualisés du Net, on découvre une nouvelle forme de tourisme sexuel : le tourisme sexuel virtuel.

\section{Les usages $d$ 'Internet à des fins de tourisme sexuel}

Thaillande, Cambodge, Chine, Tunisie, Maroc, Cuba, Colombie, Costa Rica, Brésil, République dominicaine, Russie, Tchécoslovaquie. Voilà autant de destinations qui créent des résonances chez des touristes à la recherche d'expériences sexuelles nouvelles et exotiques. Le tourisme, quand il se présente comme une expérience qui permet d'aller à la rencontre de l'Autre et de sa culture, est ancré dans les représentations que l'on se fait de l'Autre comme exotique, résolument étranger et authentique. On peut attribuer l'augmentation du nombre de touristes dans le monde à une certaine « démocratisation économique » du voyage. Le tourisme serait aussi un phénomène associé à la modernité qui définit le sujet moderne comme un sujet nécessairement en mouvement (Urry, 1995). Ainsi, à l'instar de Wang (2000), ce touriste-sujet s'actualise dans un ensemble de pratiques caractéristiques de la modernité : participation à un système de production des commodités, invention des loisirs liés à l'institutionnalisation des temps libres et à la nécessité de rompre avec la monotonie du quotidien, etc. Cet ensemble de facteurs, qui permet d'expliquer « objectivement » l'intérêt pour le voyage, 


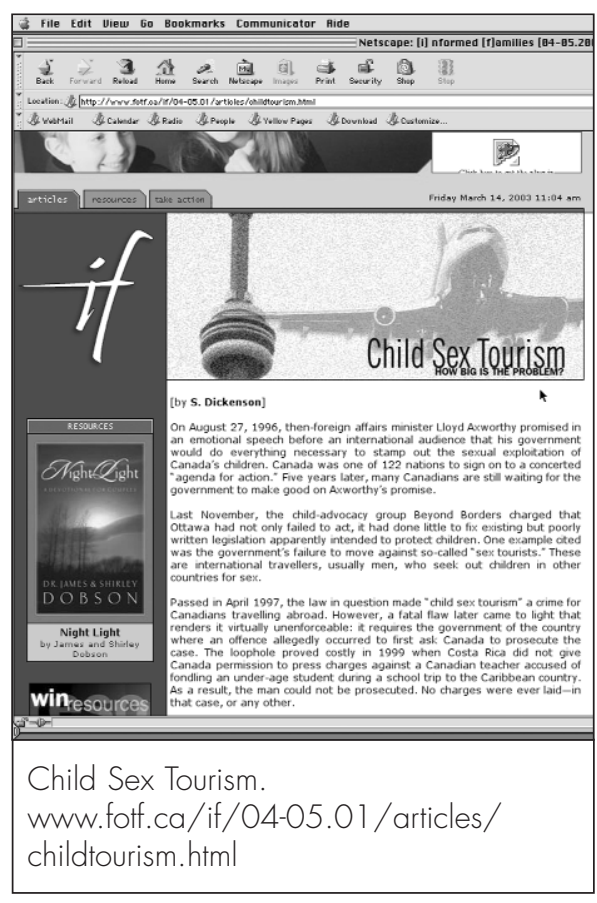

fait cependant l'économie d'une analyse des lieux visités en tant qu'ils sont des constructions sociales. Ainsi, « la destination » existe comme réalité à travers les représentations, les discours et les pratiques qui la constituent comme lieu réel. À ce titre, on peut penser que le tourisme sexuel se trouve aux frontières de ces réalités sociologiques $^{1}$, ce que nous aborderons à la prochaine section.

\section{Internet et la construction du tourisme sexuel}

Le recours à Internet s'impose aujourd'hui dans le domaine de la planification des voyages qui était, il n'y a pas très longtemps, monopolisé par les guides et les récits de voyage traditionnels ${ }^{2}$. En comparaison aux médiums traditionnels, les guides de voyage sur Internet offrent un contenu beaucoup plus diversifié, dynamique et constamment actualisé. Certains guides populaires suivent aussi cette tendance et sont maintenant disponibles en version électronique sur Internet ${ }^{3}$; ils proposent, en plus du contenu traditionnel, des forums de discussion et des récits de voyage qui permettent aux usagers de suivre au jour le jour les aventures de certains collaborateurs.

À cette transformation dans le champ de l'information relative à la planification des voyages, Internet a permis à une nouvelle presse spécialisée, notamment sur le plan sexuel, de s'afficher. Comme le soulignent Calis et Salvaggio (2002), ce phénomène n'est pas très surprenant puisque la plupart des technologies de communication ont rapidement été, du téléphone à Internet, détournées de leurs fonctions initiales vers des utilisations à connotation sexuelle. Cela s'avère tout autant pour le tourisme puisqu' on répertorie aujourd'hui plusieurs centaines de sites consacrés à la présentation de la sexualité comme dimension inhérente au voyage.

L'extension d'Internet dans les mondes contemporains permet à quiconque de construire un site Internet pour y diffuser de l'information. Aussi, les moteurs de recherche actuels sont si performants qu'ils répertorient même des sites mineurs, ce qui contribue à la prolifération de l'exposition de ces sites dédiés au tourisme sexuel. Il faut noter que si plusieurs sites visent la dénonciation de ce type de tourisme, particulièrement dans le cas de l'exploitation des enfants ${ }^{4}$, d'autres se constituent comme des outils essentiels pour la planification de la sexualité dans le contexte du voyage. À ces outils s'ajoutent des récits sexuels qui réfèrent aux espaces sexualisés du tourisme dans divers pays. Ces récits participent à la construction symbolique d'un Éden, d'un Paradis, d'une terre des milles plaisirs où l'authenticité se trouve dans la consommation sexuelle de l'autre : le tourisme sexuel devient anthropophage.

Nous analyserons à la prochaine section certains contenus proposés sur des sites Internet axés sur le tourisme sexuel.

\section{La commercialisation de la sexualité dans le tourisme sur Internet}

Avec les outils de recherche puissants maintenant disponibles sur Internet, il n'est plus nécessaire d'être un expert en informatique pour découvrir de l'information, même sur des thèmes très spécialisés. Une recherche simple nous a permis d'identifier de nombreux sites Internet dédiés à la promotion du tourisme sexuel. Des sites commerciaux, comme Dexterhorn.com, ne se cachent derrière aucune façade pour faire la promotion de ce type de tourisme. Déjà, la page d'accueil du site annonce que la Thaïlande n'est rien de moins que le Disneyland des adultes.

The land of milk and honey, Thailand is known worldwide as the «Disneyland for adults» (Dexterhorn.com [http://www.dexterhorn.com/trips/ tourthai.htm]).

La Thailande s'offrirait donc, à l'image de Disneyland, comme le lieu des excès, du rêve et du jeu. La Thaillande devient ici un espace magique fantasmé où l'expression de la sexualité est un jeu pratiqué hors du temps et surtout hors des normes qui régissent l'expression sexuelle dans le « vrai monde ». À Disneyland, les guides et les amuseurs publics donnent l'illusion d'un monde fantastique en rupture avec le réel. En Thaillande, les guides permettent au voyageur de découvrir les meilleurs sex-clubs et d'en apprendre plus qu'il n'en faut sur les coutumes et la culture de la sexualité.

They'll provide STEP by STEP instructions and guidance helping you maximize your Thailand tour of time and pleasure. Showing you the best Thailand sex clubs, restaurants, sightseeing and sex entertainment plus explaining the many Thai sex customs and cultures (Dexterhorn.com [http://www.dexterhorn.com/trips/ tourthai.htm]).

Sur le plan des guides de voyage pour célibataires en quête d'aventures sexuelles, Travel \& the Single Male ${ }^{5}$ est une référence incontournable. On annonce sur la page d'accueil que les responsables du site n'organisent aucun voyage, mais qu'ils sont plutôt des éditeurs responsables au service des hommes célibataires indépendants.

We are a responsible media travel publisher dedicated to the independent single male. We gather our information (we are «not» a travel or tour company) from many sources including our own reviewers and writers (Texte de présentation du site Internet Travel \& the Single Male [http://64.27.106.222/mirror/index2. html]). 


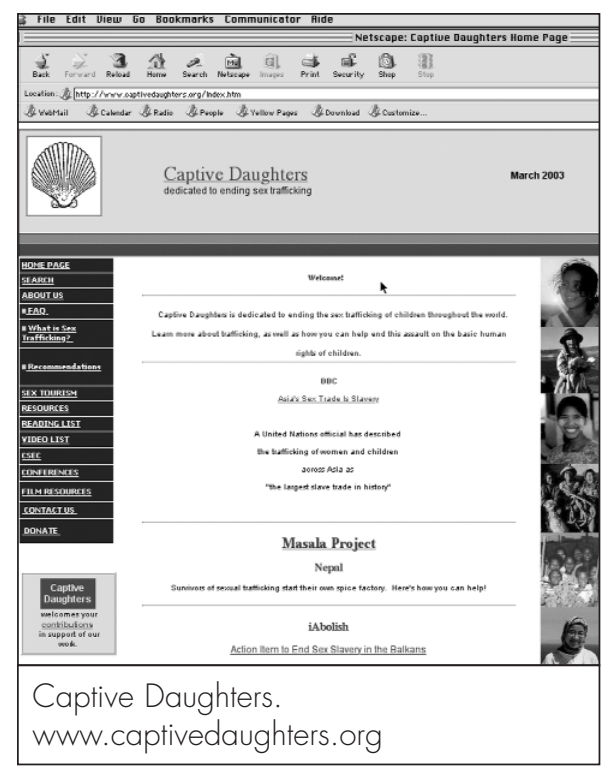

C'est sur un ton quasi journalistique qu'ils défendent leur « mission » : rapporter et informer sans parti pris.

Although adult travel can be considered risqué by some people, we feel (as any good reporter) obligated to tell and show all. We do not publish any material that is illegal, offensive, abusive, pornographic, lewd or sexually degrading. We work closely with the legitimate \& concerned adult orientated clubs of the world in correctness, promotions and discounts. Our goal is to inform \& report unbiased on all, not to offend, embarrass or ridicule (Ibid.).

Ils présentent le tourisme en célibataire comme une activité, qui peut être de bon goût, permettant d'aller au-delà du rêve et du fantasme. Le nombre important de membres, les récits de voyage et les photos qui les accompagnent, participent à faire du tourisme sexuel une réalité pour le voyageur célibataire en quête d'expériences sexuelles exceptionnelles.

Yes, adult (single) travel is a reality (and can be done in good taste). Why look or dream when you can have «all» you want - it is possible! Over 6,000 members worldwide with over 3000 articles and 1,000,s of real photos of the women our members have encountered (good, bad and yes, the ugly) (Ibid.).
Les pays y sont présentés par une mise en exergue de certaines dimensions socioculturelles qui fondent leur exotisme, sinon leur érotisme. À cet égard, le site Internet Travel $\&$ the Single Male propose que Phuket est « érotique », que Cuba est « espiègle », que Tahiti est «topless », que la Chine est « émergente », que Cebu, aux Philippines, est « corrompue », que Bogota est l'endroit rêvé pour repérer une épouse et que la Tunisie est tout simplement « inoubliable ». Limiter la complexité culturelle de l'Autre à un qualificatif unique, franchement sexualisé, montre à quel point le tourisme sexuel se fonde sur une logique de réduction. La culture est souvent réduite à une attitude sexuelle qui participe à assurer son authenticité.

\section{Le tourisme sexuel raconté : les communautés virtuelles}

Les communautés virtuelles sont, pour Rheingold (1994), des noyaux sociaux qui émergent d'Internet quand un nombre assez important de personnes partageant les mêmes intérêts entretiennent des relations soutenues dans le cyberespace. Il ajoute que nous communiquons dans cet espace comme dans l'espace « réel », à la différence que les rapports sont médiatisés par l'ordinateur. Il faut donc retenir ici que l'élément central du concept de communauté est la communication. Il existe une multitude de communautés virtuelles regroupées sous la bannière du tourisme. Nous retiendrons ici quelques sites qui présentent un intérêt pour mieux comprendre les liens entre tourisme, Internet et sexualité.

Les forums de discussion représentent une source riche d'information sur les constructions du tourisme sexuel. Les textes consultés abordent des thèmes aussi variés que les meilleurs et les pires pays pour rencontrer une compagne pour une relation à long ou à court terme, les risques de contracter une IST (infection sexuellement transmissible) et les moyens de s'en prémunir, les actes sexuels pratiqués et les tarifs exigés.

Des interventions sur le forum de discussion WSG (World Sex Guide) révèlent l'étendue de l'expérience de tourisme sexuel de certains membres. L'extrait suivant illustre à quel point le rapport aux au- tres cultures renvoie à une logique de surenchère liée à la consommation sexuelle :

The escorts I have picked up either in well known bars, high class hotels or by phoning an agency. I have kept sort of track of every girl (professionals and non-profs) I have met from 1958 onwards 265 girls in the following countries: USA, Canada, Mexico, Brazil, Argentine, Chile, New Zealand, Australia, the Philippines, Singapore, Hong Kong, Japan, Korea, Ireland, Great Britain, Denmark, Norway, Finland, Sweden, Russia, Estonia, Poland, Czech Republic, Germany, Belgium, Holland, France, Italy, Switzerland, Turkey, Hungary. That makes a selection of 32 countries. That is the base I have for determining the countries I like the best (Posté le 17 octobre 2001 sur [www.WSGforum.com]).

Pour d'autres participants, l'attrait pour les femmes de certains pays repose sur des attitudes sexuelles, la pratique de certains actes sexuels comme la sodomie et la fellation sans condom et sur les tarifs établis pour divers services sexuels. Ces critères tendent à uniformiser l'expérience sexuelle des femmes au sein d'un même pays et à occulter les conditions dans lesquelles elles pratiquent leur travail du sexe. Aussi, la référence à certaines pratiques sexuelles plus risquées en regard à la transmission des IST ou du VIH/sida suggère-t-elle que les touristes sexuels s'exposent à des risques importants qui compromettent leur santé sexuelle et celle des femmes avec qui ils ont des rapports sexuels.

Morocco: Strengths: Beauty, price, attitude, availability; Weaknesses: Privates are usually unkempt, anal.

Cameroon: Strengths: Beauty, anal, price, attitude, availability, privates; Weaknesses: Variety (95\% Negroid).

Côte d'Ivoire: Strengths: Beauty, price, attitude, variety, availability, privates; Weaknesses: Anal, BBBJ6.

Kenya: Strengths: Beauty, price, attitude, anal, BBBJ, availability; Weaknesses: Variety (90\% Negroid), privates. 
Madagascar: Strengths : Beauty, price, attitude, BBBJ; Weaknesses: Availability, anal, privates, availability.

Senegal: Strengths : Beauty, price, attitude, availability; Weaknesses: Anal, BBBJ, privates [...] (Posté le 10 mai 2002 sur [www.WSGforum.com]).

Les forums de discussion sont aussi utilisés pour demander des conseils précis sur la planification d'un voyage à des fins sexuelles.

I'm gearing up to spend the sex holiday of a life time this year. I've done UK, Holland, Germany, Eire, Canada and Dominican Rep in the past. All were good, in their own way, but I'm looking for something a bit more exotic/risky/FUN. I'm interested in knowing what is the new frontier in the world of sex? (Posté le 4 janvier 2002 sur [www.WSGforum.com]).

Le prochain extrait, posté par un membre de World Sex Guide, illustre bien le malaise de certains hommes face aux gains réalisés par les femmes en matière d'équité socio-sexuelle.

Maybe one reason why women from the USA have such bad attitudes is because of Feminism which teaches them to be wary of men and which puts men in a very bad light. These feminists do nothing the whole day long but complain about everything from gender inequality (although it seems to me that men seem to be the ones being discriminated against in current times), to complaints about the laws and so on. Then when the woman you have fallen in love with from a third world country gets into the USA, she gets corrupted by these feminists influence and you wonder why her attitude changes. Maybe men should start to speak up against feminism and expose it for what it really is, instead of keeping quiet (Posté le 8 décembre 2001 sur [www.WSGforum.com]).

Ici, le féminisme est pointé comme un moteur du tourisme sexuel et les femmes rencontrées dans d'autres cultures sont idéalisées. On les considère très différen- tes des femmes occidentales qui seraient, depuis la dernière vague féministe, trop peu ouvertes aux réels besoins sexuels des hommes (hétérosexuels). L'authenticité de l'expérience se retrouve dans le fait que les femmes rencontrées en voyage partagent leur culture d'une façon toute particulière, à travers le corps et la sexualité. Le récit de voyage se confond ici au récit sexuel où les rapports avec les autres femmes sont considérés comme un moyen d'accès privilégié à la culture.

Les avis et les conseils des voyageurs expérimentés destinés aux novices constituent une autre dimension importante des sites Internet dédiés au tourisme sexuel. Dans l'extrait suivant, tiré de la liste de discussion du site Xenono's Big Sex Travel Adventure 7 , la sexualité dans le contexte du tourisme est délimitée par les discours des voyageurs d'expérience. On expose ici le cadre qui permet de se représenter l'expérience avant même que la rencontre avec l'autre ait eu lieu.

Tsm newbies read first - It doesn't matter if you first destination is Cuba, Boca Chica, PI, etc. Jetsouth's experiences are worth noting. I think many of us more experienced travelers are just used to the drill. Our reports reflect what we like and don't like, but the "what to expect» is sometimes lacking as it is old hat to us (Tiré du site Internet Xenono's Big Sex Travel Adventure [http://xenono. clubhombre.com]).

Les extraits présentés ici ne sont bien entendu qu'une sélection, assez aléatoire, des nombreux textes consultés sur les forums de discussion axés sur le tourisme sexuel. De ceux-ci, on retient que les adeptes du tourisme sexuel, qu'ils soient expérimentés ou novices, entretiennent une position plutôt conservatrice par rapport à la sexualité. Ils mettent en évidence les conceptions occidentales de la sexualité et de la culture dans le contexte du tourisme. La sexualité, le plus souvent dans sa forme hétérosexuelle et pénétrative, est le moyen par lequel l'homo occidentalis moderne continue de coloniser l'Autre. Dans cette logique d'accumulation, il y a peu de place pour penser les rapports de pouvoir qui traversent les rencontres sexuelles entre le touriste et les tra- vailleuses du sexe, ce que certaines recherches mettent bien en évidence (Hall, 1994).

Révéler ses aventures sexuelles dans un récit de voyage (ou sur un forum de discussion) c'est, conformément à ce que Foucault (1976) a bien montré dans son histoire de la sexualité, l'extension à Internet d'une tendance déjà amorcée depuis longtemps en Occident qui fait de la sexualité une réalité de plus en plus dépendante de sa mise en discours. Révéler sa sexualité et surtout les histoires qui s'y rattachent permet aux individus de se situer dans l'ordre du discours (Plummer, 1995) et de donner un sens à leur expérience.

Cet article a permis de mettre en évidence l'extension du phénomène du tourisme sexuel à Internet. Il suggère qu' on assiste à une amplification textuelle du phénomène (plus de gens en parlent), ce qui permet de croire à une véritable augmentation du nombre de touristes intéressés par l'attrait strictement sexuel du voyage. Cette réflexion nous invite à considérer les dimensions socio-historiques complexes liées à la construction du voyage comme expérience sexualisée et Internet devient, dès lors, un médium pour mieux la saisir. Entre les discours, les échanges, la navigation et le «surfing », Internet propose une nouvelle géographie que certains parcourent touristiquement et sexuellement. Avec Internet, nous sommes obligés de repenser nos conceptions du tourisme et de ses rapports à la culture et à la sexualité de l'Autre. Il nous faut y intégrer la virtualité, la déterritorialisation et réfléchir sur les nouvelles modalités sur lesquelles s'établissent les rapports aux autres.

\section{Conclusion : le tourisme sexuel dans le cyberespace}

Le cyberespace transforme les modes de sociabilité sexuelle en permettant à l'usager de jouer avec son identité socio-sexuelle, en éliminant les marqueurs de genre, en déstabilisant les modes hiérarchiques de relations et en dispersant l'usager dans le temps et dans l'espace, à la différence des relations en face-à-face. De ce fait, le cyberespace ouvre des horizons originaux liés à l'expression de la diversité sexuelle et à l'ex- 
ploration de nouvelles formes de pratiques et d'imaginaires. Pour certains, la configuration du cyberespace contribuerait à une « nouvelle révolution sexuelle » (Schneider, 2000 ; Cooper et al. 1999).

Bien peu de travaux ont été réalisés sur les effets des multiples usages d'Internet sur la formation et l'organisation des réseaux socio-sexuels. Ce nouvel espace présente des caractéristiques qui nous obligent à repenser les rapports à l'espace, au temps et à la circulation propre au voyage et au tourisme. En d'autres termes, nous aimerions poser ici l'hypothèse que si le cyberespace se constitue comme un nouveau monde dont la géographie complexe se démarque de celle du monde « réel », on peut envisager que certaines pratiques, comme le tourisme, s'y retrouvent, mais sous une forme nouvelle. Naviguer dans Internet c'est transformer la perspective de ceux qui voyagent. Le navigateur reste désormais assis devant son écran qui se transforme pour le faire voyager de Montréal à Bangkok, puis vers Paris, New York, Rio de Janeiro et Moscou. Il n'y a plus de transit, plus d'attente et le temps requis pour aller de Montréal à Tananarive est, objectivement et à quelques secondes près, le même que celui qui mène de Montréal à Québec. Les « clics » remplacent les décollages et les atterrissages et les destinations se dévoilent parfois au hasard puisqu'elles sont peu planifiées ou prévisibles. On se rend de moins en moins à une destination précise. Plutôt, on « tombe sur un site $»$ ! Naviguer dans Internet devient une métaphore du tourisme (Nakamura, 1999).

En préparant cet article, je suis « tombé » sur plusieurs sites qui m'ont fait imaginer ma propre démarche comme un voyage. Le rapport à Internet comme lieu de rencontre avec l'altérité transforme clairement notre rapport au temps et à l'espace. Sur le Net, nous ne nous déplaçons plus vers l'Autre, mais faisons venir l'Autre vers nous. Cette métaphore de la navigation comme tourisme met en relief la complexité des phénomènes de communication et les manières par lesquelles nous cherchons constamment à nous approprier ce qui nous est extérieur pour mieux nous comprendre et nous définir : peut-on penser au-delà de l'anthropophagie ?
Lovis-Robert Frigault est professeur au Département de sexologie de l'UQAM. L'auteur aimerait souligner la collaboration de Alexandre Houle, Karine Charron et Gabrielle Beauregard à la préparation de cet article.

\section{Notes}

1 Nous ferons ici l'économie d'une histoire (ou des histoires) du tourisme. L'ouvrage de Wang (2000) propose à cet égard une analyse très fine des dimensions historiques et sociologiques du tourisme.

2 On peut penser ici au rôle de certains ouvrages publiés sur papier qui accompagnent les voyageurs dans leurs découvertes d'un nouveau pays et de ses richesses historiques, culturelles, architecturales, etc.

3 Par exemple, Lonely Planet [http://www. lonelyplanet.com] et GoTravel [http:// www.letsgo.com/] proposent des versions électroniques de leurs publications.

4 The ZeroChild sex tourism [http:// www.vachss.com/help_text/sex_tourism. html] ; Captive Daugthers [http://www. captivedaughters.org/Index.htm] ; European Commission [http://europa.eu.int /comm/enterprise/services/tourism/ policy areas /child.htm] ; [http://www.thefuture group. org/youwillbecaught/] ; [http:// www.fotf.ca/if/0405.01/articles/child tourism.html] ; [http:// parl.gc.ca/bills /government/C-27/C-27_3/C-27_ cover-E.html].

5 Travel \& The Single Male est un site Internet qui propose à ses membres un éventail de conseils pour maximiser les rencontres amoureuses ou sexuelles dans un vaste nombre de pays. Fondé sur l'échange d'information entre les 6000 utilisateurs membres du site Internet, il annonce sur la page réservée aux non-membres qu'on n'y trouve pas moins de 4000 photographies, 3500 articles couvrant 73 pays, un site de discussion en direct et un babillard électronique [http://www.tsmtravel.com/].

6 BBBJ (bareback blow-job), expression souvent rencontrée sur les forums de discussion qui signifie une fellation sans condom.

7 Xenono's Big Sex Travel Adventure [http://xenono.clubhombre.com].

\section{Bibliographie}

Calis, Luc, et Salvino A. Salvaggio (2002),

" Rencontres et sexualité sur l'Internet »,
Anthropologie de la société digitale - Tome 2, Bruxelles, Haute École Groupe ICHEC - Institut Supérieur de Commerce Saint-Louis - ISFSC.

Cooper, Alvin, Coralie R. Scherer, Sylvain C. Boies, et Barry L. Gordon (1999), « Sexuality on the Internet: From Sexual Exploration to Pathological Expression », Professional Psychology: Research and Practice, vol. 30, p. 154-164.

Foucault, Michel (1976), Histoire de la sexualité. La volonté de savoir. Paris, Gallimard.

Hall, Michael C. (1994), «Gender and Economic Interests in Tourism Prostitution: The Nature, Development and Implications of Sex Tourism in South-East Asia », dans Vivian Kinnaird et Derek Hall (dir.), Tourism. A Gender Analysis, Chichester, John Wiley \& Sons, p. 142-163.

Hughes, Donna M. (1996), « Sex Tours Via the Internet », Agenda: A Journal about Women and Gender (South Africa), vol. 28, p. 71-76.

Nakamura, Lisa (1999), « Race In/For Cyberspace: Identity Tourism and Racial Passing on the Internet », dans Victor J. Vitanza (dir.), CyberReader, 2e édition, Needham Heights, MA, Allyn \& Bacon, p. 442-451.

NETendances (2003), Sondage CEFRIO Léger Marketing 2003 [http://www.infometre. cefrio.qc.ca/loupe/omnibus/default.asp], consulté le 24 janvier 2003.

Plummer, Kenneth (1995), Telling Sexual Stories, London, Routledge.

Poster, Mark (1990), The Mode of Information, Chicago, University of Chicago Press.

Rheingold, Howard (1994), « A Slice of Life in my Virtual Community », dans L.M. Harasim (dir.) Global Networks: Computers and International Communication, Cambridge, MIT Press, p. 57-80.

Schneider, Jennifer. P. (2000), « A Qualitative Study of Cybersex Participants: Gender Differences, Recovery Issues, and Implications for Therapists ", Sexual Addiction \& Compulsivity, vol. 7, p. 249-278.

UNICEF (2001), Profiting from Sexual Abuse, [http://www.unicef.org/pubsgen/profiting/ profiting.pdf].

Urry, John (1995), Consuming Places. London, Routledge.

Wang, Ning (2000), Tourism and Modernity, New York, Pergamon. 